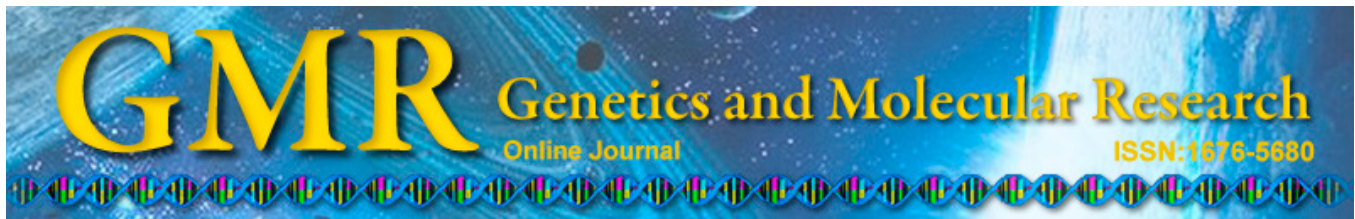

\title{
Genetic structure and genetic diversity of single-variety Lonicera macranthoides populations in China, as indicated by SCoT markers
}

\author{
D.X. Chen ${ }^{1,2,3,4}$ L.Y. Li ${ }^{1,2,3,4}$ X. Zhang ${ }^{1,2,3,4}$ and Y. Wang ${ }^{1,2,3,4}$ \\ ${ }^{1}$ Chongqing Academy of Chinese Materia Medica, Chongqing, China \\ ${ }^{2}$ Chongqing Engineering Research Center for Fine Variety Breeding \\ Techniques of Chinese Materia Medica, Chongqing, China \\ ${ }^{3}$ Chongqing Key Laboratory of Chinese Medicine Resources, Chongqing, China \\ ${ }^{4}$ Chongqing Sub-Centers of The National Resource Center for Chinese Materia \\ Medica, China Academy of Chinese Medical Sciences, Chongqing, China \\ Corresponding author: L.Y. Li \\ E-mail: lilongyun8@163.com
}

Genet. Mol. Res. 14 (3): 8058-8067 (2015)

Received November 12, 2014

Accepted April 6, 2015

Published July 17, 2015

DOI http://dx.doi.org/10.4238/2015.July.17.14

\begin{abstract}
Lonicera macranthoides is an important traditional Chinese herb. The lack of information regarding the genetic structure and genetic relationships among its cultivars has hindered the conservation and utilization of this resource. This study used start codon targeted markers to assess the genetic diversity and other genetic characteristics of five single-variety L. macranthoides populations in China. Using 22 primers produced a total of 266 bands, of which 227 were polymorphic, indicating a high level of polymorphism. At the species level, genetic diversity was high: percentage of polymorphic loci $(P P B)=85.34 \%$, effective number of alleles $\left(N_{\mathrm{E}}\right)=1.3479$, Nei's gene diversity $(H)=$ 0.2075 , and Shannon's information index $\left(H_{\mathrm{sp}}\right.$, species level $)=0.3198$. However, at the varietal population level, genetic diversity was lower,
\end{abstract}


with averages of: $P P B=19.74 \%, N_{\mathrm{E}}=1.0946, H=0.0561, H_{\text {pop }}=0.0850$ (population level). Nei's genetic differentiation coefficient was 0.7319 , which is consistent with Shannon's population genetic differentiation coefficient (0.7324). This indicates that most of the genetic variation in this species exists among the varietal populations. The differentiation among varieties may have been caused by artificial selection, mode of reproduction, and barriers to gene flow (0.1831). The genetic similarity coefficient ranged from 0.7222 to 0.9419 . Phylogenetic analysis showed the five varieties to form two major clades. Results suggest that cultivar breeders should strengthen the exchange of germplasm and increase the mutual penetration of useful genes, which would broaden the hereditary basis of L. macranthoides.

Key words: Lonicera macranthoides; Genetic diversity; Genetic structure; SCoT

\section{INTRODUCTION}

Lonicera macranthoides is a semi-evergreen perennial vine of the Caprifoliaceae family. It is an economically relevant crop used for both medicinal and ornamental purposes. It was first listed as one of the base source plants of Flos Lonicerae in the 2005 edition of Chinese Pharmacopoeia volume I (Chinese Pharmacopoeia Committee, 2005). The dried flower buds and early-opening flowers of this plant have medicinal applications. $L$. macranthoides contains flavonoids, volatile oils, organic acids, saponins, and other chemical constituents (Mao et al., 1993; Chen et al., 2012c). These compounds have shown efficacy against pathogenic microorganisms, fever, hepatic maladies, and other health problems (Wang et al., 2009a; Guan et al., 2011). L. macranthoides has been used to treat pharyngitis, furuncles and carbuncles, erysipelas, heat toxins, blood dysentery, the common cold, febrile disease, and fever. This plant is widely cultivated in Hunan, Chongqing, Sichuan, and Guizhou provinces and in some areas of Hubei, Anhui, Zhejiang, Guangxi, Jiangxi, and Yunnan provinces. At present, the main source of $L$. macranthoides herb is artificial cultivation, which has a 1500year history in China. Both the applied and basic research associated with the cultivation of this plant are relatively mature (Li et al., 2010; Zhang et al., 2010; Chu et al., 2011). Studies have fostered some success in the breeding of fine varieties (Wang et al., 2004; Wang and Huang, 2004). Because the genetic backgrounds and genetic relationships between cultivated varieties and wild germplasm sources are unclear, cross-breeding has not been reported. To evaluate germplasm genetic diversity, studies have been carried out using a variety of molecular markers (Wang et al., 2009b; Chen et al., 2011, 2012a). However, there is no information regarding genetic variation in L. macranthoides at the single-variety population level. Reported studies have illustrated genetic variation using two-dimensional analysis based on genetic distance and the genetic similarity coefficient. Evaluations of the genetic structure of the inner multi-dimensional relationship between the materials used in breeding, especially hybrid parent affinity and selection, are limited by the scant amount of reference material. One of the key problems in the breeding of L. macranthoides is the limited understanding of the genetic structure of its varieties. The genetic relationships among varietal populations must be described explicitly to guide parental selection in crossbreeding. 
Start codon targeted polymorphism (SCoT), a method developed by Collard and Mackill (2009), is a new target molecular marker technology. Its single primers are based on the flanking sequences of the ATG translation initiation site in plant genes and are able to amplify multiple regions of the genome. They are also versatile and can be used in different species. The amplification products are separated and detected using simple agarose gel electrophoresis. This technology has the advantages of easy operation, low cost, and high amplification efficiency, as well as abundant detectable polymorphisms. At present, this molecular marker technology has been applied to a variety of plants to detect genetic diversity or differential gene expression (Collard and Mackill, 2009; Wu et al., 2010; Xiong et al., 2011; Guo et al., 2012). It has also been used with medicinal plants (Chen et al., 2012b). In this study, we used SCoT molecular marker technology to analyze the genetic structure of five L. macranthoides varietal populations (Table 1). The purpose of this study was to provide a theoretical basis for the rational use and scientific protection of resources.

\section{MATERIAL AND METHODS}

\section{Plant materials}

Individuals (86) were selected from five L. macranthoides varieties based on the degree of cracking of the corolla (Table 1). Their genetic diversity and genetic structure were analyzed. The L. macranthoides varieties used in this study were preserved in a Chongqing Xiushan germplasm nursery. Plants were sampled during the flowering stage. After identification of each variety, we took samples using the principles of uniform distribution and random sampling, attempting to cover the range of cultivated varieties. Individual plants (20 per variety) were collected and fresh, young, unopened buds were stored at low temperatures for extraction of genomic DNA.

Table 1. Materials used in this study.
\begin{tabular}{llcl}
\hline No. & Variety name & Sample size & \\
\hline CG & Changgui & 17 & Corolla at flowering stage \\
BY & Baiyun & 16 & Fully open \\
YL & Yulei 1 & 18 & Semi-open \\
JCL & Jingchuilei & 18 & Closed \\
XL & Xianglei 1 & 17 & Closed \\
\hline
\end{tabular}

\section{Extraction and detection of genomic DNA}

Plant DNA was prepared using a genomic DNA extraction kit (Tiangen, Beijing, China). DNA quality and quantity were measured using a spectrophotometer (Smart $\mathrm{Spec}^{\mathrm{TM}}$ 3000, Bio-Rad, Hercules, CA, USA) and 1\% agarose gel electrophoresis, respectively.

\section{SCoT-PCR amplification and detection}

Using previously published and reported sequences (Collard and Mackill, 2009), SCoT primers were synthesized by the Shanghai Sangon Biological Engineering Co., Ltd. (China). Thirty-six SCoT primers were used to screen two randomly selected DNA templates from each population. The names of selected primers and their sequences are shown in Table 2. 
PCR was performed in a $15-\mu \mathrm{L}$ reaction mixture containing $1 \mathrm{X}$ PCR buffer, $1.5 \mathrm{mM}$ $\mathrm{Mg}^{2+}, 200 \mu \mathrm{M}$ dNTP, $0.4 \mu \mathrm{M}$ primers, 40 ng DNA, $1 \mathrm{U}$ Taq DNA polymerase. The PCR program consisted of one cycle at $94^{\circ} \mathrm{C}$ for $1 \mathrm{~min} ; 36$ cycles at $94^{\circ} \mathrm{C}$ for $1 \mathrm{~min}$ and at $50^{\circ} \mathrm{C}$ for 1 min, $72^{\circ} \mathrm{C}$ for $2 \mathrm{~min}$; and a final extension step at $72^{\circ} \mathrm{C}$ for $10 \mathrm{~min}$. The products were stored at $4^{\circ} \mathrm{C}$. Amplification was performed in $\mathrm{S} 1000^{\mathrm{TM}}$ Thermal Cycler (Bio-Rad) on 96-well plates.

Amplification products $(5 \mu \mathrm{L})$ were mixed with $1 \mu \mathrm{L} 6 \mathrm{X}$ DNA loading buffer containing nucleic acid dye. The mixture was analyzed by gel electrophoresis on $1.5 \%$ agarose gel with $1 \mathrm{X}$ TAE electrophoresis buffer at $150 \mathrm{~V}$ for about $1 \mathrm{~h}$. Gels were visualized and recorded using an automated imaging system (Gel Doc XR, Bio-Rad).

\section{Statistical analysis}

The gel electrophoresis results of the PCR amplification products showed clear, stable, and easy-to-identify DNA bands. The presence or absence of bands was translated into a binary data matrix. For each allele, the presence of a band was recorded as "1" and its absence was recorded as " 0 ".

The populations used in this study were assumed to be in Hardy-Weinberg equilibrium. The results of the PCR amplification were analyzed for genetic parameters using the Popgene 1.31 software. These parameters included total genetic diversity $\left(H_{t}\right)$, genetic diversity within populations $\left(H_{\mathrm{s}}\right)$, genetic differentiation coefficient $\left(G_{\mathrm{ST}}\right)$, gene flow $\left(N_{\mathrm{m}}\right)$, Nei's genetic distance $(D)$, genetic identity $(I)$, percentage of polymorphic loci $(P P B)$, Nei's gene diversity $(H)$, observed number of alleles $\left(N_{\mathrm{A}}\right)$, effective number of alleles $\left(N_{\mathrm{E}}\right)$, and Shannon's information index $\left(H_{\mathrm{O}}\right) . H_{\mathrm{O}}$ was calculated at two levels: total diversity $\left(H_{\mathrm{sp}}\right.$, species level $)$ and the average diversity within each population ( $H_{\text {pop }}$, population level). We estimated the genetic variation among populations using Shannon's population differentiation factor (similar to $G_{\mathrm{ST}}$ ), derived from the following equation:

$$
\left(H_{\mathrm{sp}}-H_{\mathrm{pop}}\right) / H_{\mathrm{sp}}
$$

(Equation 1)

A dendrogram was generated using unweighted pair group method with arithmetic mean (UPGMA) in order to analyze the evolutionary relationships among populations.

\section{RESULTS}

\section{Polymorphism samplified by SCoT primers}

In order to evaluate the genetic differences among the five L. macranthoides varietal populations, we detected polymorphisms for SCoT bands among 86 samples, using SCoT primers. Ultimately, 22 primers were found to produce clear and repeatable amplification products. These showed relatively high rates of polymorphism and were selected for further amplification. The results are shown in Table 2. A total of 266 bands were produced and 227 of these were polymorphic. The number of amplified bands per primer ranged from 6 to 20 , with an average of 12.1. Of these, an average of 10.3 loci were polymorphic. The polymorphic percentage ranged between 63.64 and $100 \%$. This showed the efficiency of SCoT markers to be high with respect to the detection of polymorphisms. 
Table 2. Sequences and PCR results using SCoT primers.

\begin{tabular}{|c|c|c|c|c|}
\hline Primer & Sequences & Total bands & Polymorphic bands & Polymorphic bands (\%) \\
\hline SP3 & CAACAATGGCTACCACCG & 10 & 8 & 80.00 \\
\hline SP4 & CAACAATGGCTACCACCT & 9 & 7 & 77.78 \\
\hline SP5 & CAACAATGGCTACCACGA & 6 & 5 & 83.33 \\
\hline SP6 & CAACAATGGCTACCACGC & 11 & 10 & 90.91 \\
\hline SP7 & CAACAATGGCTACCACGG & 9 & 6 & 66.67 \\
\hline SP8 & CAACAATGGCTACCACGT & 13 & 11 & 84.62 \\
\hline SP9 & CAACAATGGCTACCAGCA & 12 & 12 & 100.00 \\
\hline SP11 & AAGCAATGGCTACCACCA & 12 & 10 & 83.33 \\
\hline SP12 & ACGACATGGCGACCAACG & 16 & 16 & 100.00 \\
\hline SP13 & ACGACATGGCGACCATCG & 11 & 10 & 90.91 \\
\hline SP15 & ACGACATGGCGACCGCGA & 20 & 20 & 100.00 \\
\hline SP16 & ACCATGGCTACCACCGAC & 10 & 8 & 80.00 \\
\hline SP17 & ACCATGGCTACCACCGAG & 14 & 12 & 85.71 \\
\hline SP21 & ACGACATGGCGACCCACA & 11 & 9 & 81.82 \\
\hline SP23 & CACCATGGCTACCACCAG & 11 & 7 & 63.64 \\
\hline SP25 & ACCATGGCTACCACCGGG & 9 & 8 & 88.89 \\
\hline SP29 & CCATGGCTACCACCGGCC & 13 & 9 & 69.23 \\
\hline SP30 & CCATGGCTACCACCGGCG & 17 & 15 & 88.24 \\
\hline SP31 & CCATGGCTACCACCGCCT & 9 & 7 & 77.78 \\
\hline SP33 & CCATGGCTACCACCGCAG & 18 & 15 & 83.33 \\
\hline SP34 & ACCATGGCTACCACCGCA & 15 & 13 & 86.67 \\
\hline SP36 & GCAACAATGGCTACCACC & 10 & 9 & 90.00 \\
\hline Total & & 266 & 227 & 85.34 \\
\hline Average & & 12.1 & 10.3 & \\
\hline
\end{tabular}

\section{Genetic diversity of varietal populations}

We calculated the associated genetic parameters of the five varieties at the species and population levels (Table 3). At the species level, genetic diversity was as follows: $P P B=$ $85.34 \%, N_{\mathrm{E}}=1.3479, H=0.2075$, and $H_{\mathrm{sp}}=0.3198$. At the varietal population level, the averages for the genetic parameters examined were as follows: $P P B=19.74 \%, N_{\mathrm{E}}=1.0946, \mathrm{H}=$ $0.0561, H_{\text {pop }}=0.085$. The following results were obtained for bud-type varieties, at the species level: $P P B=52.63 \%, N_{\mathrm{E}}=1.2658, H=0.1543, H_{\mathrm{sp}}=0.2315$; at the varietal population level: $P P B=11.28 \%, N_{\mathrm{E}}=1.0471, H=0.0283, H_{\mathrm{pop}}=0.0443$.

Table 3. Genetic variation of five Lonicera macranthoides varietal populations.

\begin{tabular}{|c|c|c|c|c|c|c|c|}
\hline Variety & $\mathrm{N}$ & $K$ & $P P B(\%)$ & $N_{\mathrm{A}}$ & $N_{\mathrm{E}}$ & $H$ & $H_{\mathrm{O}}$ \\
\hline CG & 17 & 98 & 36.84 & 1.3684 & 1.1723 & 0.1040 & 0.1606 \\
\hline BY & 16 & 71 & 26.69 & 1.2669 & 1.1594 & 0.0913 & 0.1360 \\
\hline YL & 18 & 30 & 11.28 & 1.1128 & 1.0377 & 0.0238 & 0.0387 \\
\hline JCL & 18 & 42 & 15.79 & 1.1579 & 1.0736 & 0.0430 & 0.0660 \\
\hline $\mathrm{XL}$ & 17 & 18 & 6.77 & 1.0677 & 1.0301 & 0.0182 & 0.0283 \\
\hline Average & & 52 & 19.74 & 1.1947 & 1.0946 & 0.0561 & 0.0859 \\
\hline Species level & 86 & 227 & 85.34 & 1.8534 & 1.3479 & 0.2075 & 0.3198 \\
\hline
\end{tabular}

$\mathrm{N}=$ sample sizes; $K=$ polymorphic loci; $P P B=$ percentage of polymorphic bands; $N_{\mathrm{A}}=$ observed number of alleles; $N_{\mathrm{E}}=$ effective number of alleles; $H=$ Nei's genetic diversity; $H_{\mathrm{O}}=$ Shannon's information index $\left(H_{\mathrm{pop}}\right.$ : at the varietal population level, $H_{\mathrm{sp}}$ : at the species level).

The five varieties showed abundant genetic diversity, while the bud-type varieties showed only moderate diversity. At the varietal population level, genetic diversity among the populations was low but differences between them were significant. The flowering-type variety 
CG showed the highest diversity, followed by the semi-flowering-type variety BY and the three bud-type varieties, in that order. The results of four genetic parameters $\left(P P B, N_{\mathrm{E}}, H, H_{\mathrm{O}}\right)$ indicated that variety CG showed the most genetic variation, followed by BY, JCL, YL, and XL.

\section{Genetic differentiation and gene flow}

Under the assumption of genetic equilibrium, the $H_{\mathrm{t}}$ of the five varietal populations was 0.2090 , the $H_{\mathrm{s}}$ was 0.0560 , and the genetic diversity among varietal populations $\left(D_{\mathrm{ST}}=\right.$ $H_{\mathrm{t}}-H_{\mathrm{s}}$ ) was 0.1530 (Table 3 ). $G_{\mathrm{ST}}$ was 0.7319 , indicating a genetic variation of $73.19 \%$ among populations and $26.81 \%$ within populations.

$H_{\mathrm{O}}$ was used to measure diversity at the species level $\left(H_{\mathrm{sp}}=0.3198\right)$ and at the varietal population level $\left(H_{\text {pop }}=0.0850\right.$; Table 4$)$. Based on these data, we calculated the Shannon's population differentiation coefficient $\left(H_{\mathrm{sp}}-H_{\mathrm{pop}}\right) / H_{\mathrm{sp}}$ to be 0.7324 , showing $73.24 \%$ of the genetic variation to be distributed among the populations and $26.58 \%$ within the populations.

\begin{tabular}{|c|c|c|c|c|c|}
\hline & CG & BY & YL & JCL & XL \\
\hline CG & - & 0.8536 & 0.8232 & 0.8061 & 0.7533 \\
\hline BY & 0.1583 & - & 0.7480 & 0.7706 & 0.8150 \\
\hline YL & 0.1945 & 0.2904 & - & 0.9419 & 0.7222 \\
\hline JCL & 0.2156 & 0.2606 & 0.0598 & - & 0.7464 \\
\hline $\mathrm{XL}$ & 0.2833 & 0.2045 & 0.3255 & 0.2925 & - \\
\hline
\end{tabular}

The results of analysis of these two kind methods were almost identical, showing genetic differentiation to be greater among populations than within populations. This indicates that inter-varietal variation is the main source of diversity in varietal populations of $L$. macranthoides.

$N_{\mathrm{m}}$ is the movement of genes among and within populations and is negatively correlated with their genetic differentiation (Grant, 1991). It is important for the mobility and evolution of plant populations and can be calculated using the following formula (McDermott and McDonald, 1993):

$$
N_{\mathrm{m}}=0.5\left(1-G_{\mathrm{ST}}\right) / G_{\mathrm{ST}}
$$

Wright (1951) reported that $N_{\mathrm{m}}$ values greater than 1 indicate that gene flow is occurring between populations. In the present study, $N_{\mathrm{m}}$ among varietal populations of $L$. macranthoides was 0.1831 , indicating a low level of $N_{\mathrm{m}}$ and a high degree of genetic differentiation. $N_{\mathrm{m}}$ among bud-type varieties populations was even lower, at 0.110 .

\section{Genetic distance and cluster analysis}

$I$ and $D$ are shown in Table 4. $I$ ranged from 0.7222 to 0.9419 , with an average of 0.7980 . $D$ ranged from 0.0598 to 0.3255 , with an average of 0.2285 . $D$ was longest between YL and XL $(D=0.8981)$, indicating a distant phylogenetic relationship between these two 
varieties. $D$ was shortest $(D=0.0143)$ between $\mathrm{YL}$ and JCL, indicating a close phylogenetic relationship between them. Similarly for the bud-type varieties, XL was shown to be genetically distant from YL and JCL, but genetically close to BY (semi-flowering-type).

In order to reveal the genetic relationships among the five L. macranthoides varieties, based on Nei's genetic distance, a dendrogram showing their phylogenetic relationships was constructed using UPGMA (Figure 1). The resulting tree shows that the YL and JCL varietal populations cluster together (Ia), and the CG and BY varietal populations also cluster with each other (Ib). These two pairs together form a larger clade (I) that excludes XL. Due to the greater genetic distance between XL and the other varietal populations, this variety alone forms clade II.

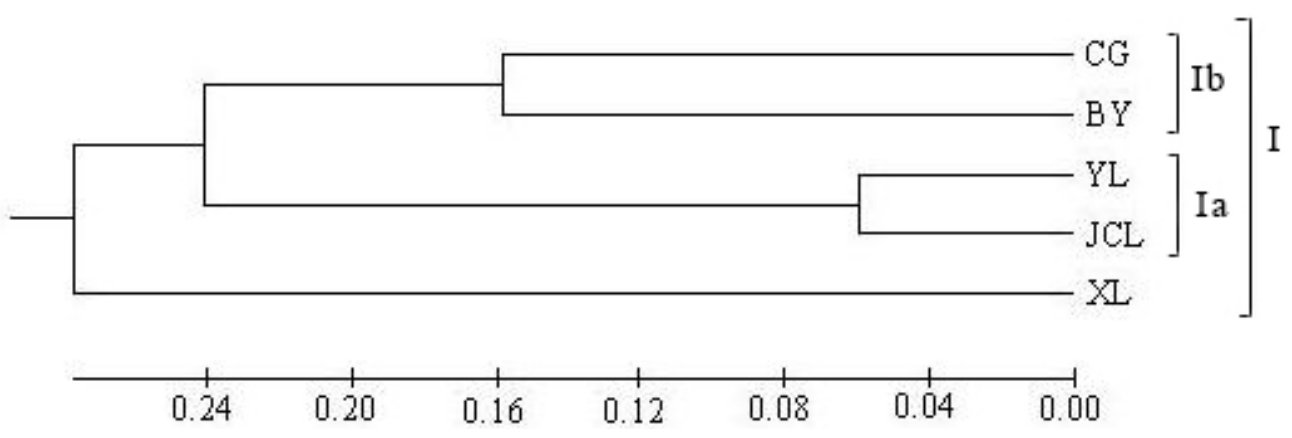

Figure 1. UPGMA dendrogram for Lonicera macranthoides varietal populations based on Nei's genetic distance $(D)$.

\section{DISCUSSION}

\section{Genetic diversity}

In recent years, genetic diversity has been investigated in both wild and cultivated germplasms of L. macranthoides. Previous literature and the results of this study both show the polymorphic loci ratio of populations of cultivated varieties to be lower than those of wild populations. This may be explained by directional selection for certain traits leading to the loss of rare alleles in the process of cultivar breeding, thereby reducing the level of genetic diversity. Cultivars that produce offspring through long-term asexual reproduction foster a relatively narrow genetic background. This, to some extent, increasing homozygosity of the germplasm.

Although the materials reported in the literature were all cultivated germplasm, the type and number of varieties, selected markers, and polymorphic efficiency of primers all differed (Wang et al., 2009b; Chen et al., 2012a). In order to indicate the real detection efficiency and consistency between inter-simple sequence repeat (ISSR) and sequence-related amplified polymorphism (SRAP) markers, Chen et al. (2012a) selected primers based on their ability to amplify the polymorphic band, rather than primers rich in polymorphisms. Wang et al. (2009b) reported the polymorphic ratio of 17 cultivated materials was $82.04 \%$ using ISSR markers, but Chen et al. (2012a) revealed the polymorphic ratio of five cultivars, using ISSR and SRAP markers, to be in the upper middle levels. In this study, the genetic diversity of five L. macranthoides varieties was analyzed using SCoT markers. The results showed that, at the 
species level, $P P B$ was $85.34 \%$, but at the varietal level, the average $P P B$ was only $19.74 \%$. L. macranthoides single-variety populations maintained a high degree of genetic diversity at the species level, consistent with their biological characteristics: First, L. macranthoides has high ecological adaptability and is widely distributed in China. In general, widely distributed species tend to maintain high levels of genetic variation. Second, L. macranthoides is a crosspollinated plant, and these also generally maintain high levels of genetic variation.

Although the varieties evaluated in this study were the same as those reported in the literature, the latter's DNA template was a mixed gene pool and determined genetic relationships using two-dimensional analysis based on genetic distances and genetic similarity coefficients. This type of analysis cannot reveal the genetic structure of varieties and cannot be applied to initial judgments of genetic diversity. In this study, individual genomic DNA served as the amplification template. This reflected genetic diversity both among and within the varietal populations and the genetic structure of inner multi-dimensional relationship. This finding may guide hybrid parent selection in breeding.

\section{Genetic differentiation and $N_{\mathrm{m}}$}

Genetic differentiation and $N_{\mathrm{m}}$ are important evaluative indices of population genetic structure. In this study, we analyzed the genetic structure of five single-variety populations of L. macranthoides. Results indicate that genetic exchange between varieties has been severely hampered and that existing varieties show a high degree of genetic differentiation. Genetic variation was mainly derived among varieties. The relationship within varieties was much closer. Hamrick and Godt (1989) report that the formation of a population's genetic structure is affected by various factors, such as breeding systems, $N_{\mathrm{m}}$, seed dispersal mechanisms, natural selection, and other factors. For $L$. macranthoides, the main factors affecting genetic structure may be $N_{\mathrm{m}}$, propagation method, and artificial selection.

Plant $N_{\mathrm{m}}$ among and within natural populations is achieved by means of carriers of genetic material, such as pollen, seeds, spores, and vegetative elements (Hamrick, 1987). Of these, the spread of pollen and seeds is the most important. In the materials tested in this study, the corollas of bud-type varieties were in the closed state throughout the flowering period, preventing the spread of their own pollen and the entry of external pollen. In this condition, the spontaneous self-pollination seed rate is zero (Sun et al., 2013). $N_{\mathrm{m}}$ mediated by pollen and seeds is severely hampered. For this reason, the bud-type varieties can only be cultivated by grafting, cutting, layering, tissue culture, and other asexual propagation modes. The corollas of flowering-type and semi-flowering-type varieties remain open during the flowering period. These plants can accept external pollen from visiting insects. Under natural conditions, the seed-setting rate is $54.20 \%$; with artificial cross-pollination, the seed-setting rate increases to $86.80 \%$ (Sun et al., 2013). Flowering-type and semi-flowering-type varieties have a mixed reproductive strategy. They can be used as seeds in sexual reproduction and in asexual techniques such as grafting, cutting, and layering. Seed propagation is generally only used in the breeding of new varieties, while asexual propagation is used in most production processes. Therefore, $N_{\mathrm{m}}$ mediated by pollen and seeds is also severely hampered in flowering-type and semi-flowering-type varieties. In short, barriers to gene flow lead to significant genetic differentiation between varietal populations. Long-term asexual reproduction causes increasing homozygosity of the germplasm to some extent, inevitably narrowing the genetic background of the variety. 
The influence of artificial selection on genetic structure mainly affects the directional selection of certain traits during the variety breeding process. Current varieties are mostly focused on traits that affect yield. This focuses attention on a small number of phenotypes and decreases the diversity of other breeding targets. For this reason, in the breeding process, we should actively introduce high-quality genes for disease resistance to identify high-quality germplasm resources and broaden the genetic base of the germplasm.

\section{Conservation of genetic diversity in L. macranthoides breeding}

The key characteristics of bud-type varieties are a corolla that does not crack at any point during flowering and a long flowering period that allows only one harvest, which is convenient for production. In current production methods, flowering-type varieties are slowly being replaced by bud-type varieties. However, the genetic diversity of flowering-type and semi-flowering-type varieties is significantly higher than that of bud-type varieties. Floweringtype varieties also exhibit abundant genetic variation, indicating significant potential for the breeding of new varieties and the genetic improvement of existing varieties. Because of their diminished tendency to crack, bud-type varieties can increase the vulnerability and singleness of populations, indicating an extremely narrow genetic basis. In order to develop new varieties rich in genetic diversity, full advantage should be taken of existing local germplasm resources and attention should be paid to preserving the genetic diversity of various types, including flowering-type and semi-flowering-types in local and farm varieties. Doing so would provide the material foundation for the future breeding of new varieties. If these varieties cannot be effectively protected or are artificially eliminated, the genetic diversity of cultivated varieties may be greatly reduced. For this reason, these varieties should be given special protection.

China is rich in wild L. macranthoides with a high degree of variability in important phenotypes, such as the length of the flowering period, the timing of flowering, the density of flower clusters, the openness of the corolla, and disease resistance. This variation is valuable for its potential use in cultivar breeding. For this reason, the genes of wild germplasm should be evaluated continuously. This may permit the breeding of new varieties and the improvement of existing varieties.

Because L. macranthoides has high genetic diversity among varieties and low genetic diversity within varieties, it may benefit from the establishment of germplasm resource gardens. Attention should be paid to sampling strategies, which can increase the genetic diversity of cultivars. Briefly, we suggest sampling as many varieties as possible and ensuring that the number of individuals from any one variety is not too large. Attention should also be paid to strengthening the exchange of breed germplasm, which increases the mutual penetration of high-quality genes.

L. macranthoides has a long history of cultivation and a wide distribution in China. The five cultivars evaluated in this study alone cannot represent the genetic background of all L. macranthoides cultivars. The genetic structure and genetic diversity of $L$. macranthoides cultivars require further study. In order to foster an in-depth understanding of the resource situation regarding these cultivars and to provide a basis for the exploration and rational use of China's rich L. macranthoides genetic resources, follow-up studies are needed to test more samples. The samples must be collected across a wide area to adequately cover the area of cultivation. 


\section{ACKNOWLEDGMENTS}

Research supported by the National Science and Technology Support Program of China (\#2006BAI06A12-14), the Natural Science Foundation of Chongqing (\#CSTC2009BB5395), the National Science and Technology Benefiting Plan (\#2013GS500102), and the Construction Plan for Science and Technology platform and base of Chongqing (\#cstc2014ptyjd10001).

\section{REFERENCES}

Chen DX, Li LY, Wu YK, Zhang X, et al. (2011). Genetic diversity of Lonicera macranthoides geographical populations revealed by SRAP markers. Chin. Tradit. Herbal. Drugs 42: 143-147.

Chen DX, Zhang X and Li LY (2012a). Analysis of genetic diversity and genetic relationships of Lonicera macranthoides cultivars. Chin. J. Chin. Mater. Med. 37: 1912-1916.

Chen DX, Zhang X, Wang Y and LiL Y (2012b). Genetic diversity of Scrophularia ningpoensis based on SCoT analysis. Chin. J. Chin. Mater. Med. 37: 2368-2372.

Chen Y, Zhao YY, Wu S, Wang M, et al. (2012c). Water-soluble chemical constituents in flower buds of Lonicera macranthoides. Chin. Med. Mat. 35: 231-234.

Chinese Pharmacopoeia Committee (2005). Chinese Pharmacopoeia (Volume I). The Chemical Industry Press, Beijing, China, 21-22, 152-153.

Chu C, Liu HJ, Qi LW, Liu EH, et al. (2011). Combination of normal light and fluorescence microscopy for authentication of five Lonicera species flower buds. Microsc. Res. Tech. 74: 133-141.

Collard BCY and Mackill DJ (2009). Start codon targeted (SCoT) polymorphism: A simple, novel DNA marker technique for generating gene-targeted markers in plants. Plant Mol. Biol. Rep. 27: 86-93.

Grant V (1991). The evolutionary process: A critical study of evolutionary theory. Columbia University Press, New York.

Guan FQ, Shan Y, Zhao XZ, Zhang DM, et al. (2011).Apoptosis and membrane permeabilisation induced by macranthoside B on HL-60 cells. Nat. Prod. Res. 25: 332-340.

Guo DL, Zhang JY and Liu CH (2012). Genetic diversity in some grape varieties revealed by SCoT analyses. Mol. Biol. Rep. 39: 5307-5313.

Hamrick JL (1987). Gene flow distribution of genetic variation in plant populations. In: Differentiation patterns in higher plants (Urbanska KM, ed.). Academic Press, New York, 53-67.

Hamrick JL and Godt MJW (1989). Allozyme diversity in plant species. In: Plant population genetics, breeding and genetic resources (Brown AHD, Clegg MT, Kahler AL and Weir BS, eds.). Sinauer Press, Sunderland, MA, 43-63.

Li LY, Zhang X and Yang X (2010). Anatomical study on rooting of Lonicera macranthoides cutting. China J. Chin. Mat. Med. 35: 431-434.

Mao Q, Cao D and Jia XS (1993). Studies on the chemical constituents of Lonicera macranthoides Hand.-Mazz. Acta Pharm. Sin. 28: 273-281.

McDermott JM and McDonald BA (1993). Gene flow in plant pathosystems. Annu. Rev. Phytopathol. 31: 353-373.

Sun NX, Li LY and Cui GL (2013). Study on flowering characteristics and breeding system of Lonicera macranthoides Hand.-Mazz. Southwest China J. Agric. Sci. 26: 1178-1183.

Wang J, Zhao XZ, Qi Q, Tao L, et al. (2009a). Macranthoside B, a hederageninsaponin extracted from Lonicera macranthoides and its anti-tumor activities in vitro and in vivo. Food Chem. Toxicol. 47: 1716-21.

Wang S, Zhou RB, Pan QP, Liu XD, et al. (2009b). Genetic diversity analysis of Lonicera macranthoides Hand.-Mazz. germplasm by ISSR markers. Pharm. Biotechnol. 16: 149-152.

Wang XM, Luo JD, Song QA, Ma SJ, et al. (2004). Selective breeding of new cultivars of Lonicera macranthoides Hand.Mazz. Hunan Forest. Sci. Technol. 31: 15-17.

Wang Z and Huang Y (2004). Preliminary report on the breeding and promotion of Xianglei honeysuckle. Chin. Med. Mat. 27: 896-897.

Wright S (1951). The genetical structure of populations. Ann. Eugen. 15: 323-354.

Wu JM, Li YR, Wang AQ, Yang L, et al. (2010). Differential expression of gibberellin-induced genes for stalk elongation of sugarcane analyzed with cDNA-SCoT. Acta Agronom. Sin. 36: 1883-189.

Xiong F, Zhong R, Han Z, Jiang J, et al. (2011). Start codon targeted polymorphism for evaluation of functional genetic variation and relationships in cultivated peanut (Arachis hypogaea L.) genotypes. Mol. Biol. Rep. 38: 3487-3494.

Zhang X, Li LY and Yang X (2010). Nutrient change in Lonicera macranthoides during cutting propagation process. China J. Chin. Mat. Med. 35: 1378-1381. 